\title{
On Fuzzy Ordered Abel-Grassmann's Groupoids
}

\author{
Madad Khan \& Faisal \\ Department of Mathematics \\ COMSATS Institute of Information Technology \\ Abbottabad, Pakistan \\ E-mail: madadmath@yahoo.com,yousafzaimath@yahoo.com
}

Received: December 31, 2010 Accepted: January 21, 2011 doi:10.5539/jmr.v3n2p27

\begin{abstract}
In this paper, we have introduced the concept of fuzzy ordered AG-groupoids which is the generalization of fuzzy ordered semigroups first considered by Kehayopulu and Tsingelis (2002). We have studied some important features of a left regular ordered AG-groupoid in terms of fuzzy left ideals, fuzzy right ideals, fuzzy two-sided ideals, fuzzy generalized bi-ideals, fuzzy bi-ideals, fuzzy interior ideals and fuzzy $(1,2)$-ideals. We have shown that the set of all fuzzy two-sided ideals of a left regular ordered AG-groupoid forms a semilattice structure. We have characterized all the fuzzy ideals of a left regular ordered AG-groupoid. Finally we have characterized a left regular ordered AG-groupoid by their fuzzy left and fuzzy right ideals.
\end{abstract}

Keywords: Ordered AG-groupoids, Left regular ordered AG-groupoids, Left invertive law and fuzzy ideals

\section{Introduction}

The concept of fuzzy sets was first proposed by Zadeh in 1965, which has a wide range of applications in various fields such as computer engineering, artificial intelligence, control engineering, operation research, management science, robotics and many more. It gives us a tool to model the uncertainty present in a phenomena that do not have sharp boundaries. Many papers on fuzzy sets have been appeared which shows the importance and its applications to set theory, algebra, real analysis, measure theory and topology etc. (see Chang, C. L. 1968; Kuroki, N. 1979; and Rosenfeld, A. 1971).

An Abel Grassmann's groupoid, abbreviated as an AG-groupoid is a groupoid $S$ whose elements satisfy the left invertive law $(a b) c=(c b) a$, for all $a, b, c \in S$. The concept of this algebraic structure was first introduced by Kazim and Naseeruddin in 1972 and they have called it a left almost semigroup (LA-semigroup). In an AG-groupoid, the medial law (Kazim, M. A. 1972) $(a b)(c d)=(a c)(b d)$ holds for all $a, b, c, d \in S$. An AG-groupoid may or may not contains a left identity. The left identity of an LA-semigroup allow us to introduce the inverses of elements in an AG-groupoid. If an AG-groupoid contains a left identity, then it is unique (Mushtaq, Q., 1978). In an AG-groupoid $S$ with left identity, the paramedial law $(a b)(c d)=(d c)(b a)$ holds for all $a, b, c, d \in S$. If an AG-groupoid contains a left identity, then by using medial law, we get $a(b c)=b(a c)$, for all $a, b, c \in S$. Several examples and interesting properties of AG-groupoids can be found in (Mushtaq, Q., 1978) and (Protic, 1994).

An AG-groupoid is a non-associative and non-commutative algebraic structure mid way between a groupoid and a commutative semigroup. This structure is closely related with a commutative semigroup, because if an AG-groupoid contains a right identity, then it becomes a commutative semigroup (Mushtaq, Q., 1978). The connection of a commutative inverse semigroup with an AG-groupoid has been given in (Mushtaq, Q. 1988) as, a commutative inverse semigroup ( $S, \circ$ ) becomes an AG-groupoid $(S, \cdot)$ under $a \cdot b=b \circ a^{-1}$, for all $a, b \in S$. An AG-groupoid $S$ with left identity becomes a semigroup under the binary operation "o" defined as, if for all $x, y \in S$, there exists $a \in S$ such that $x \circ y=(x a) y$ (Protic, 1994). An AG-groupoid is the generalization of a semigroup theory (Mushtaq, Q. 1978) and has vast applications in collaboration with semigroup like other branches of mathematics.

The connection of AG-groupoids with the vector spaces over finite fields has been investigated as: Let $W$ be a sub-space of a vector space $V$ over a field $F$ of cardinal $p^{n}$ for some prime $p \neq 2$. Define the binary operation " $\odot$ " on $W$ as follows: $u \odot v=\alpha^{\frac{p^{n}-1}{2}} u+v$, where $\alpha$ is a generator of $F \backslash\{0\}$ and $u, v \in W$. Then $(W, \odot)$ is an AG-groupoid with left identity 0 .

From the above discussion, we see that AG-groupoids have very closed links with semigroups and vector spaces which shows the importance and applications of AG-groupoids.

An ordered AG-groupoid (po-AG-groupoid) is a structure $(S, ., \leq)$ in which the following conditions hold.

(i) $(S,$.$) is an AG-groupoid.$

(ii) $(S, \leq)$ is a poset (reflexive, anti-symmetric and transitive).

(iii) For all $a, b$ and $x \in S, a \leq b$ implies $a x \leq b x$ and $x a \leq x b$. 
Example 1 Consider an open interval $\mathbb{R}_{\mathbb{O}}=(0,1)$ of real numbers under the binary operation of multiplication. Define $a * b=b a^{-1} r^{-1}$, for all $a, b, r \in \mathbb{R}_{\mathbb{O}}$, then it is easy to see that $\left(\mathbb{R}_{\mathbb{O}}, *, \leq\right)$ is an ordered AG-groupoid under the usual order $" \leq$ " and we have called it a real ordered AG-groupoid.

Throughout in this paper $S$ will be considered as an ordered AG-groupoid unless otherwise specified.

\section{Preliminaries}

In this section, we have given some basic definitions which are necessary for the subsequent sections.

A fuzzy subset or a fuzzy set of a non-empty set $S$ is an arbitrary mapping $f: S \rightarrow[0,1]$, where $[0,1]$ is the unit segment of real line. A fuzzy subset $f$ is a class of objects with a grades of membership having the form $f=\{(s, f(s)) / s \in S\}$.

Let $x \in S$, then $A_{x}=\{(y, z) \in S \times S \backslash x \leq y z\}$.

The product of any fuzzy subsets $f$ and $g$ of $S$ is defined by

$$
(f \circ g)(x)=\left\{\begin{array}{c}
\bigvee_{(y, z) \in A_{x}}\{f(y) \wedge g(z)\} \text { if } x \leq y z=\bigvee_{x \leq y z}\{f(y) \wedge g(z)\} \text { if } A_{x} \neq \emptyset \\
0 \quad \text { if } A_{x}=\emptyset
\end{array}\right.
$$

The order relation $\subseteq$ between any two fuzzy subsets $f$ and $g$ of $S$ is defined by

$$
f \subseteq g \text { if and only if } f(x) \leq g(x) \text {, for all } x \in S .
$$

The symbols $f \cap g$ and $f \cup g$ will mean the following fuzzy subsets of $S$

$$
(f \cap g)(x)=\min \{f(x), g(x)\}=f(x) \wedge g(x), \text { for all } x \text { in } S,
$$

and

$$
(f \cup g)(x)=\max \{f(x), g(x)\}=f(x) \vee g(x), \text { for all } x \text { in } \mathcal{S} \text {. }
$$

For $\emptyset \neq A \subseteq S$, we define

$$
(A]=\{t \in S \mid t \leq a, \text { for some } a \in A\} .
$$

For $A=\{a\}$, we usually written as $(a]$.

A subset $A$ of $S$ is called semiprime if $a^{2} \in A$ implies $a \in A$. A fuzzy subset $f$ of $S$ is called a fuzzy semiprime if $f(a) \geq f\left(a^{2}\right)$ for all $a$ in $S$.

A non-empty subset $A$ of an $S$ is called a left (right) ideal of $S$ if

(i) $S A \subseteq A(A S \subseteq A)$.

(ii) If $a \in A$ and $b$ is in $S$ such that $b \leq a$, then $b$ is in $A$.

A subset $A$ of $S$ is called a two-sided ideal of $S$ if it is both a left and a right ideal of $S$.

A fuzzy subset $f$ of $S$ is called a fuzzy left (right) ideal of $S$ if

(i) $x \leq y \Rightarrow f(x) \geq f(y)$.

(ii) $f(a b) \geq f(b)(f(a b) \geq f(a))$, for all $a$ and $b$ in $S$.

A fuzzy subset $f$ of $S$ is called a fuzzy two-sided ideal of $S$ if it is both a fuzzy left and a fuzzy right ideal of $S$.

A fuzzy subset $f$ of $S$ is called a fuzzy AG-subgroupoid of $S$ if $f(a b) \geq f(a) \wedge f(b)$, for all $a$ and $b$ in $S$.

A fuzzy subset $f$ of $S$ is called a fuzzy generalized bi-ideal of $S$ if

(i) $x \leq y \Rightarrow f(x) \geq f(y)$.

(ii) $f((x y) z) \geq f(x) \wedge f(z)$, for all $x, y$ and $z$ in $S$.

A fuzzy AG-subgroupoid $f$ of $S$ is called a fuzzy bi-ideal of $S$ if

(i) $x \leq y \Rightarrow f(x) \geq f(y)$.

(ii) $f((x y) z) \geq f(x) \wedge f(z)$, for all $x, y$ and $z$ in $S$.

A fuzzy subset $f$ of $S$ is called a fuzzy interior ideal of $S$ if

(i) $x \leq y \Rightarrow f(x) \geq f(y)$.

(ii) $f((x y) z) \geq f(y)$, for all $x, y$ and $z$ in $S$. 
A fuzzy subset $f$ of $S$ is called a fuzzy idempotent if $f \circ f=f$.

For $\emptyset \neq A \subseteq S$, the characteristic function, $C_{A}$ is defined by

$$
C_{A}= \begin{cases}1 & \text { if } x \in A, \\ 0 & \text { if } x \notin A .\end{cases}
$$

Note that $S$ can be considered as a fuzzy subset of itself and we write $S=\mathbf{C}_{S}$, i.e. $S(x)=1$, for all $x \in S$.

Example 2 Let $S=\{a, b, c\}$ be an ordered AG-groupoid defined by the following multiplication and order below.

$$
\begin{array}{r}
\cdot \\
\begin{array}{r|rrr}
a & b & c \\
\hline a & a & a & a \\
b & a & a & c \\
c & a & a & a
\end{array} \\
\leq:=\{(a, a),(b, b),(c, c),(a, c),(a, b)\}
\end{array}
$$

Define a fuzzy subset $f$ of $S$ as follows: $f(a)=0.8, f(b)=0.4$ and $f(c)=0.6$, then it is easy to see that $f$ is a fuzzy two-sided ideal of $S$.

Note that every fuzzy two-sided ideal of $S$ is a fuzzy AG-subgroupoid of $S$ but the converse is not true in general. For this, let us define a fuzzy subset $f$ of $S$ as follows: $f(a)=0.9, f(b)=0.7$ and $f(c)=0.5$, then one can easily observe that $f$ is a fuzzy AG-subgroupoid of $S$ but $f$ is not a fuzzy two-sided ideal of $S$, because $f(b c) \nsupseteq f(b)$.

\section{Fuzzy ordered AG-groupoids in terms of fuzzy subsets}

We denote by $F(S)$ the set of all fuzzy subsets of $S$.

Proposition 1. The set $(F(S), \circ, \subseteq)$ is an ordered $A G$-groupoid.

Proof. Clearly $F(S)$ is closed. Let $f, g$ and $h$ be in $F(S)$. If $A_{x}=\emptyset$ for any $x \in S$, then $((f \circ g) \circ h)(x)=0=((h \circ g) \circ f)(x)$. Let $A_{x} \neq \emptyset$, then there exist $y$ and $z$ in $S$ such that $(y, z) \in A_{x}$. Therefore by using left invertive law, we have

$$
\begin{aligned}
((f \circ g) \circ h)(x) & =\bigvee_{(y, z) \in A_{x}}\{(f \circ g)(y) \wedge h(z)\} \\
& =\bigvee_{(y, z) \in A_{x}}\left\{\bigvee_{(p, q) \in A_{y}}\{f(p) \wedge g(q)\} \wedge h(z)\right\} \\
& =\bigvee_{x \leq(p q) z}\{f(p) \wedge g(q) \wedge h(z)\} \\
& =\bigvee_{x \leq(z q) p}\{h(z) \wedge g(q) \wedge f(p)\} \\
& =\bigvee_{(w, p) \in A_{x}}\left\{\bigvee_{(z, q) \in A_{w}}(h(z) \wedge g(q) \wedge f(p))\right\} \\
& =\bigvee_{(w, p) \in A_{x}}\{(h \circ g)(w) \wedge f(p)\}=((h \circ g) \circ f)(x) .
\end{aligned}
$$

Hence $(F(S), \circ)$ is an AG-groupoid.

Assume that $f \subseteq g$ and let $A_{x}=\emptyset$ for any $x \in S$, then $(f \circ h)(x)=0=(g \circ h)(x) \Longrightarrow f \circ h \subseteq g \circ h$ and similarly we can show that $h \circ f \subseteq h \circ g$. Let $A_{x} \neq \emptyset$, then there exist $y$ and $z$ in $S$ such that $(y, z) \in A_{x}$, therefore

$$
(f \circ h)(x)=\bigvee_{(y, z) \in A_{x}}\{f(y) \wedge h(z)\} \leq \bigvee_{(y, z) \in A_{x}}\{g(y) \wedge h(z)\}=(g \circ h)(x) .
$$

Similarly we can show that $(h \circ f)(x) \leq(h \circ g)(x)$ holds for all $x \in S$. Thus $(F(S), \circ, \subseteq)$ is an ordered AG-groupoid.

Corollary 1. The left invertive law holds in $F(S)$.

Corollary 2. The medial law holds in $F(S)$.

The following Corollary is direct consequence of the successive use of left invertive law in Proposition 1. 
Corollary 2. For any fuzzy subsets $f, g$ and $h$ of $S$, the following conditions are equivalent:

(i) $(f \circ g) \circ h=g \circ(f \circ h)$,

(ii) $(f \circ g) \circ h=g \circ(h \circ f)$.

Note that the Corollaries 1 and 2 are the analogues definitions of left invertive law and medial law in fuzzy context.

Proposition 2. An ordered AG-groupoid $S$ with $F(S)=(F(S))^{2}$ is a commutative ordered semigroup if and only if $(f \circ g) \circ h=f \circ(h \circ g)$ holds for all fuzzy subsets $f, g, h \in F(S)$.

Proof. Let $S$ be a commutative ordered semigroup. For any fuzzy subsets $f, g, h \in F(S)$, if $A_{x}=\emptyset$ for any $x \in S$, then $((f \circ g) \circ h)(x)=0=(f \circ(h \circ g))(x)$. Let $A_{x} \neq \emptyset$, then there exist $s$ and $t$ in $S$ such that $(s, t) \in A_{x}$, therefore by use of left invertive law and commutative law, we get

$$
\begin{aligned}
((f \circ g) \circ h)(x) & =\bigvee_{(s, t) \in A_{x}}\{(f \circ g)(s) \wedge h(t)\} \\
& =\bigvee_{(s, t) \in A_{x}}\left\{\bigvee_{(m, n) \in A_{s}} f(m) \wedge g(n) \wedge h(t)\right\} \\
& =\bigvee_{x \leq(m n) t}\{f(m) \wedge h(t) \wedge g(n)\} \\
& =\bigvee_{x \leq(t n) m}\{f(m) \wedge h(t) \wedge g(n)\} \\
& =\bigvee_{x \leq m(t n)}\{f(m) \wedge h(t) \wedge g(n)\} \\
& =\bigvee_{(m, p) \in A_{x}}\left\{\bigvee_{(t, n) \in A_{p}} f(m) \wedge h(t) \wedge g(n)\right\} \\
& =\bigvee_{(m, p) \in A_{x}}\{f(m) \wedge(h \circ g)(p)\}=(h \circ(f \circ g))(x) .
\end{aligned}
$$

Conversely, let $(f \circ g) \circ h=f \circ(h \circ g)$ holds for all fuzzy subsets $f, g, h \in F(S)$. We have to show that $S$ is a commutative ordered semigroup. Let $f$ and $g$ be any arbitrary fuzzy subsets of $S$, if $A_{x}=\emptyset$ for any $x \in S$, then $(f \circ g)(x)=0=(g \circ f)(x)$. Let $A_{x} \neq \emptyset$, then there exist $s$ and $t$ in $S$ such that $(s, t) \in A_{x}$. Since $F(S)=(F(S))^{2}$, so $f=h \circ k$, where $h$ and $k$ are any fuzzy subsets of $S$. Now by using left invertive law, we have

$$
\begin{aligned}
(f \circ g)(x) & =((h \circ k) \circ g)(x)=\bigvee_{(s, t) \in A_{x}}\{(h \circ k)(s) \wedge g(t)\} \\
& =\bigvee_{(s, t) \in A_{x}}\left\{\bigvee_{(m, n) \in A_{s}} h(m) \wedge k(n) \wedge g(t)\right\} \\
& =\bigvee_{x \leq(m n) t}\{g(t) \wedge k(n) \wedge h(m)\} \\
& =\bigvee_{x \leq(t n) m}\{g(t) \wedge k(n) \wedge h(m)\} \\
& =\bigvee_{(p, m) \in A_{x}}\left\{\bigvee_{(t, n) \in A_{p}} g(t) \wedge k(n) \wedge h(m)\right\} \\
& =\bigvee_{(p, m) \in A_{x}}\{(g \circ k)(p) \wedge h(m)\}=(g \circ(h \circ k))(x)
\end{aligned}
$$

This shows that $f \circ g=g \circ(h \circ k)=g \circ f$. Therefore commutative law holds in $S$.

Now if $A_{x}=\emptyset$ for any $x \in S$, then $((f \circ g) \circ k)(x)=0=(f \circ(g \circ k))(x)$. Let $A_{x} \neq \emptyset$, then there exist $s$ and $t$ in $S$ such that 
$(s, t) \in A_{x}$, therefore by use of left invertive law and commutative law, we get

$$
\begin{aligned}
((f \circ g) \circ k)(x) & =\bigvee_{(s, t) \in A_{x}}\{(f \circ g)(s) \wedge k(t)\} \\
& =\bigvee_{(s, t) \in A_{x}}\left\{\bigvee_{(m, n) \in A_{s}} f(m) \wedge g(n) \wedge k(t)\right\} \\
& =\bigvee_{x \leq(m n) t}\{f(m) \wedge g(n) \wedge k(t)\} \\
& =\bigvee_{x \leq(t n) m}\{f(m) \wedge g(n) \wedge k(t)\} \\
& =\bigvee_{x \leq m(t n)}\{f(m) \wedge g(n) \wedge k(t)\} \\
& =\bigvee_{x \leq m(n t)}\{f(m) \wedge g(n) \wedge k(t)\} \\
& =\bigvee_{(m, p) \in A_{x}}\left\{\bigvee_{(n, t) \in A_{p}} f(m) \wedge g(n) \wedge k(t)\right\} \\
& =\bigvee_{(m, p) \in A_{x}}\{f(m) \wedge(g \circ k)(p)\}=(f \circ(g \circ k)(x)
\end{aligned}
$$

Theorem 1. If $S$ has a left identity, then the following properties hold in $F(S)$.

(i) $f \circ(g \circ h)=g \circ(f \circ h)$, for all $f, g$ and $h$ in $F(S)$.

(ii) $(f \circ g) \circ(h \circ k)=(k \circ h) \circ(g \circ f)$, for all $f, g$, h and $k$ in $F(S)$.

Proof. (i) : Let $x$ be an arbitrary element of $S$. If $A_{x}=\emptyset$ for $x \in S$, then $(f \circ(g \circ h))(x)=0=(g \circ(f \circ h))(x)$. Let $A_{x} \neq \emptyset$, then there exist $y$ and $z$ in $S$ such that $(y, z) \in A_{x}$. Now by using medial law with left identity, we have

$$
\begin{aligned}
(f \circ(g \circ h))(x) & =\bigvee_{(y, z) \in A_{x}}\{f(y) \wedge(g \circ h)(z)\} \\
& =\bigvee_{(y, z) \in A_{x}}\left\{f(y) \wedge \bigvee_{(p, q) \in A_{z}}\{g(p) \wedge h(q)\}\right\} \\
& =\bigvee_{x \leq y(p q)}\{f(y) \wedge g(p) \wedge h(q)\} \\
& =\bigvee_{x \leq p(y q)}\{g(p) \wedge f(y) \wedge h(q)\} \\
& =\bigvee_{(p, w) \in A_{x}}\left\{g(p) \wedge \bigvee_{(y, q) \in A_{w}}\{f(y) \wedge h(q)\}\right\} \\
& =\bigvee_{(p, w) \in A_{x}}\{g(p) \wedge(f \circ h)(w)\}=(g \circ(f \circ h))(x) .
\end{aligned}
$$

Thus, $(f \circ(g \circ h))(x)=(g \circ(f \circ h))(x)$, for all $x$ in $S$.

(ii) : If $A_{x}=\emptyset$ for $x \in S$, then $((f \circ g) \circ(h \circ k))(x)=0=((k \circ h) \circ(g \circ f))(x)$. Let $A_{x} \neq \emptyset$, then there exist $y$ and $z$ in $S$ 
such that $(y, z) \in A_{x}$. Therefore by using paramedial law, we have

$$
\begin{aligned}
((f \circ g) \circ(h \circ k))(x) & =\bigvee_{(y, z) \in A_{x}}\{(f \circ g)(y) \wedge(h \circ k)(z)\} \\
& =\bigvee_{(y, z) \in A_{x}}\left\{\bigvee_{(p, q) \in A_{y}}\{f(p) \wedge g(q)\} \wedge \bigvee_{(u, v) \in A_{z}}\{h(u) \wedge k(v)\}\right\} \\
& =\bigvee_{x \leq(p q)(u v)}\{f(p) \wedge g(q) \wedge h(u) \wedge k(v)\} \\
& =\bigvee_{x \leq(v u)(q p)}\{k(v) \wedge h(u) \wedge g(q) \wedge f(p)\} \\
& =\bigvee_{(m, n) \in A_{x}}\left\{\bigvee_{(v, u) \in A_{m}}\{k(v) \wedge h(u)\} \wedge \bigvee_{(q, p) \in A_{n}}\{g(q) \wedge f(p)\}\right\} \\
& =\bigvee_{(m, n) \in A_{x}}\{(k \circ h)(m) \wedge(g \circ f)(n)\}=((k \circ h) \circ(g \circ f))(x) .
\end{aligned}
$$

Thus, $(f \circ g) \circ(h \circ k)=(k \circ h) \circ(g \circ f)$, for all $x$ in $S$.

Theorem 2. $Q=\{f \mid f \in F(S), f \circ h=f$, where $h=h \circ h\}$ is a commutative monoid in $S$.

Proof. The fuzzy subset $Q$ of $S$ is non-empty since $h \circ h=h$, which implies that $h$ is in $Q$. Let $f$ and $g$ be fuzzy subsets of $S$ in $Q$, then $f \circ h=f$ and $g \circ h=g$. If $A_{x}=\emptyset$ for $x \in S$, then $(f \circ g)(x)=0=((f \circ g) \circ h)(x)$. Let $A_{x} \neq \emptyset$, then there exist $y$ and $z$ in $S$ such that $(y, z) \in A_{x}$. Therefore by using medial law, we have

$$
\begin{aligned}
(f \circ g)(x) & =\bigvee_{(y, z) \in A_{x}}\{(f \circ h)(y) \wedge(g \circ h)(z)\} \\
& =\bigvee_{(y, z) \in A_{x}}\left\{\bigvee_{(p, q) \in A_{y}}\{f(p) \wedge h(q)\} \wedge \bigvee_{(u, v) \in A_{z}}\{g(u) \wedge h(v)\}\right\} \\
& =\bigvee_{x \leq(p q)(u v)}\{f(p) \wedge h(q) \wedge g(u) \wedge h(v)\} \\
& =\bigvee_{x \leq(p u)(q v)}\{f(p) \wedge g(u) \wedge h(q) \wedge h(v)\} \\
& =\bigvee_{(m, n) \in A_{x}}\left\{\bigvee_{(p, u) \in A_{m}}\{f(p) \wedge g(u)\} \wedge \bigvee_{(q, v) \in A_{n}}\{h(q) \wedge h(v)\}\right\} \\
& =\bigvee_{(m, n) \in A_{x}}\{(f \circ g)(m) \wedge(h \circ h)(n)\}=((f \circ g) \circ(h \circ h))(x)
\end{aligned}
$$

Thus $f \circ g=(f \circ g) \circ(h \circ h)=(f \circ g) \circ h$, which implies that $Q$ is closed.

Now if $A_{x}=\emptyset$ for $x \in S$, then $(f \circ g)(x)=0=(g \circ f)(x)$. Let $A_{x} \neq \emptyset$, then there exist $y$ and $z$ in $S$ such that $(y, z) \in A_{x}$. Therefore by using left invertive law, we have

$$
\begin{aligned}
(f \circ g)(x) & =\bigvee_{(y, z) \in A_{x}}\{(f \circ h)(y) \wedge g(z)\} \\
& =\bigvee_{(y, z) \in A_{x}}\left\{\bigvee_{(p, q) \in A_{y}} f(p) \wedge h(q) \wedge g(z)\right\} \\
& =\bigvee_{x \leq(p q) z}\{g(z) \wedge h(q) \wedge f(p)\} \\
& =\bigvee_{x \leq(z q) p}\{g(z) \wedge h(q) \wedge f(p)\} \\
& =\bigvee_{(t, p) \in A_{x}}\left\{\bigvee_{(z, q) \in A_{t}} g(z) \wedge h(q) \wedge f(p)\right\} \\
& =\bigvee_{(t, p) \in A_{x}}\{(g \circ h)(t) \wedge f(p)\}=((g \circ h) \circ f)(x) .
\end{aligned}
$$


Thus $f \circ g=(g \circ h) \circ f=g \circ f$, which implies that commutative law holds in $Q$ and associative law holds in $Q$ due to commutativity. Since for any fuzzy subset $f$ in $Q$, we have $f \circ h=f$ (where $h$ is fixed) implies that $h$ is a right identity in $S$ and hence an identity.

Lemma 1. If $S$ has a left identity, then $S \circ S=S$.

Proof. Every $x$ in $S$ can be written as $x \leq e x$, that is $(e, x) \in A_{x}$, where $e$ is the left identity in $S$. Therefore

$$
(S \circ S)(x)=\bigvee_{(e, x) \in A_{x}}\{S(e) \wedge S(x)\}=1=S(x) .
$$

Hence $S \circ S=S$.

\section{Left regular ordered fuzzy AG-groupoids}

An element $a$ of $S$ is called a left regular if there exists $x \in S$ such that $a \leq x a^{2}$ and $S$ is called a left regular if every element of $S$ is left regular.

The following three Lemmas have the same proof as in (Xie, X. Y. 2008).

Lemma 2. Let $f$ be a fuzzy subset of $S$, then $f$ is a fuzzy left ideal of $S$ if and only if $f$ satisfies the following.

(i) $x \leq y \Rightarrow f(x) \geq f(y)$, for all $x$ and $y$ in $S$.

(ii) $S \circ f \subseteq f$.

Lemma 3. Let $f$ be a fuzzy subset of $S$, then $f$ is a fuzzy right ideal of $S$ if and only if $f$ satisfies the following.

(i) $x \leq y \Rightarrow f(x) \geq f(y)$, for all $x$ and $y$ in $S$.

(ii) $f \circ S \subseteq f$.

Lemma 4. Let $f$ be a fuzzy subset of $S$, then $f$ is a fuzzy two-sided ideal of $S$ if and only if $f$ satisfies the following.

(i) $x \leq y \Rightarrow f(x) \geq f(y)$, for all $x$ and $y$ in $S$.

(ii) $S \circ f \subseteq f$ and $f \circ S \subseteq f$.

Lemma 5. In a left regular $S$ with left identity, $f \circ S=f$ and $S \circ f=f$ holds for every fuzzy two-sided ideal $f$ of $S$.

Proof. Let $f$ be a fuzzy two-sided ideal of $S$. For every $a \in S$ there exists $x \in S$ such that $a \leq x a^{2}$. Now by using paramedial and left invertive law, we have

$$
a \leq x a^{2}=(e x)(a a)=(a a)(x e)=((x e) a) a .
$$

Thus $(((x e) a), a) \in A_{a}$, since $A_{a} \neq \emptyset$, therefore

$$
\begin{aligned}
(f \circ S)(a) & =\bigvee_{(((x e) a), a) \in A_{a}}\{f((x e) a) \wedge S(a)\} \\
& \geq f(x a) \wedge S(a) \geq f(a) \wedge 1=f(a) .
\end{aligned}
$$

Now by using Lemma $3, f \circ S=f$.

Also

$$
\begin{aligned}
(S \circ f)(a) & =\bigvee_{(((x e) a), a) \in A_{a}}\{S((x e) a) \wedge f(a)\} \\
& \geq S((x e) a) \wedge f(a) \geq f(a) \wedge 1=f(a) .
\end{aligned}
$$

Now by using Lemma 2, $S \circ f=f$.

Corollary 3. For a left regular $S$ with left identity, $f \circ S=f$ and $S \circ f=f$ holds for every fuzzy left ideal $f$ of $S$.

Lemma 6. Let $f$ and $g$ be any fuzzy two-sided ideals of $S$ with left identity, then $f \circ g=f \cap g$. 
Proof. Assume that $f$ and $g$ are any fuzzy two-sided ideals of $S$. Since $S$ is left regular, then for every $a \in S$ there exists $x \in S$ such that $a \leq x a^{2}$. Now by using medial law with left identity, we have

$$
a \leq x a^{2}=x(a a)=a(x a)
$$

Thus $(a, x a) \in A_{a}$, since $A_{a} \neq \emptyset$, therefore

$$
\begin{aligned}
(f \circ g)(a) & =\bigvee_{(a, x a) \in A_{a}}\{f(a) \wedge g(x a)\} \geq f(a) \wedge g(x a) \\
& \geq f(a) \wedge g(a)=(f \cap g)(a) .
\end{aligned}
$$

Thus by using Lemmas 2 and 3, we get $f \circ g=f \cap g$.

Lemma 7. Every fuzzy two-sided ideal of a left regular $S$ with left identity is idempotent.

Proof. Let $f$ be a fuzzy two-sided ideal of $S$. For every $a \in S$ there exists $x \in S$ such that $a \leq x a^{2}$. From Lemma 5 , $a \leq((x e) a) a$. Thus $(((x e) a), a) \in A_{a}$, since $A_{a} \neq \emptyset$, therefore

$$
\begin{aligned}
(f \circ f)(a) & =\bigvee_{(((x e) a), a) \in A_{a}}\{f((x e) a) \wedge f(a)\} \\
& \geq f(a) \wedge f(a)=f(a) .
\end{aligned}
$$

Now by using Lemma $2, f \circ f=f$.

Corollary 4. Every fuzzy left ideal of a left regular $S$ is idempotent.

Theorem 3. The set of fuzzy two-sided ideals of a left regular $S$ with left identity forms a semilattice structure with identity $S$.

Proof. Let $\mathcal{F}_{I}$ be the set of all fuzzy two-sided ideals of a left regular $S$ and let $f, g$ and $h$ be in $\mathcal{F}_{I}$. Clearly $\mathcal{F}_{I}$ is closed and by Lemma 7, we have $f \circ f=f$. Now by using Lemma 6, we get $f \circ g=g \circ f$. Therefore by using left invertive law and commutative law, we have

$$
\begin{aligned}
(f \circ g) \circ h & =(g \circ f) \circ h=(h \circ f) \circ g=(f \circ h) \circ g \\
& =(g \circ h) \circ f=f \circ(g \circ h) .
\end{aligned}
$$

It is easy to see from Lemma 5 that $S$ is an identity in $\mathcal{F}_{I}$.

An element $a$ of $S$ is called a right regular if there exists $x \in S$ such that $a \leq a^{2} x$ and $S$ is called a right regular if every element of $S$ is right regular.

Remark 1 Let $S$ be a left regular ordered AG-groupoid with left identity, then for every a $\in S$ there exist some $x, y \in S$ such that $a \leq x a^{2}=a^{2} y$. Indeed

$$
\begin{aligned}
a & \leq x a^{2}=(e x)(a a)=(a a)(x e) \quad \text { by using paramedial law } \\
& =a^{2} y
\end{aligned}
$$

and obviously $a \leq a^{2} y=x a^{2}$ also holds. This shows that the concept of left regular and right regular coincide in ordered AG-groupoids with left identity.

Lemma 8. A fuzzy subset $f$ of $S$ is a fuzzy AG-subgroupoid of $S$ if and only if $f \circ f \subseteq f$.

Proof. It is simple.

Theorem 4. In a left regular $S$ with left identity, the following statements are equivalent.

(i) $f$ is a fuzzy bi-(generalized bi-) ideal of $S$.

(ii) $(f \circ S) \circ f=f$ and $f \circ f=f$. 
Proof. (i) $\Longrightarrow$ (ii) : Assume that $f$ is a fuzzy bi-ideal of a left regular $S$ with left identity and let $a \in S$, then there exists $x \in S$ such that $a \leq a^{2} x$. Now by using left invertive law, medial law with left identity and paramedial law, we have

$$
\begin{aligned}
a & \leq(a a) x=(x a) a=(x((a a) x)) a=((a a)(x x)) a \\
& =((x x)(a a)) a=(((a a) x) x) a=(((x a) a) x) a \\
& =(((x((a a) x)) a) x) a=((((a a)(x x)) a) x) a \\
& =((((x x)(a a)) a) x) a=\left(\left(\left(a\left(x^{2} a\right)\right) a\right) x\right) a .
\end{aligned}
$$

Thus $\left(\left(\left(\left(a\left(x^{2} a\right)\right) a\right) x\right), a\right) \in A_{a}$, since $A_{a} \neq \emptyset$, therefore

$$
\begin{aligned}
((f \circ S) \circ f)(a) & =\bigvee_{\left(\left(\left(\left(a\left(x^{2} a\right)\right) a\right) x\right), a\right) \in A_{a}}\left\{(f \circ S)\left(\left(\left(a\left(x^{2} a\right)\right) a\right) x\right) \wedge f(a)\right\} \\
& \geq \bigvee_{\left(\left(\left(a\left(x^{2} a\right)\right) a\right), x\right) \in A_{\left(\left(a\left(x^{2} a\right)\right) a\right) x}}\left\{f\left(\left(a\left(x^{2} a\right)\right) a\right) \wedge S(x)\right\} \wedge f(a) \\
& \geq f\left(\left(a\left(x^{2} a\right)\right) a\right) \wedge 1 \wedge f(a) \\
& \geq f(a) \wedge f(a) \wedge f(a)=f(a) .
\end{aligned}
$$

Now by using left invertive law, medial law with left identity and paramedial law, we have

$$
\begin{aligned}
a & \leq(a a) x=(x a) a=(x((a a) x)) a=((a a)(x x)) a \\
& =((x x)(a a)) a=\left(a\left(x^{2} a\right)\right) a .
\end{aligned}
$$

Thus $\left(\left(a\left(x^{2} a\right)\right), a\right) \in A_{a}$, since $A_{a} \neq \emptyset$, therefore

$$
\begin{aligned}
((f \circ S) \circ f)(a) & =\bigvee_{\left(\left(a\left(x^{2} a\right)\right), a\right) \in A_{a}}\left\{(f \circ S)\left(\left(a\left(x^{2} a\right)\right)\right) \wedge f(a)\right\} \\
& =\bigvee_{\left(\left(a\left(x^{2} a\right)\right), a\right) \in A_{a}}\left\{\bigvee_{\left(a,\left(x^{2} a\right)\right) \in A_{a\left(x^{2} a\right)}}\left\{f(a) \wedge S\left(x^{2} a\right)\right\}\right\} \wedge f(a) \\
& =\bigvee_{\left(\left(a\left(x^{2} a\right)\right), a\right) \in A_{a}}\left\{\bigvee_{\left(a,\left(x^{2} a\right)\right) \in A_{a\left(x^{2} a\right)}}\{f(a) \wedge 1\}\right\} \wedge f(a) \\
& =\bigvee_{\left(\left(a\left(x^{2} a\right)\right), a\right) \in A_{a}}\left\{\bigvee_{\left(a,\left(x^{2} a\right)\right) \in A_{a\left(x^{2} a\right)}} f(a)\right\} \wedge f(a) \\
& =\bigvee_{\left(\left(a\left(x^{2} a\right)\right), a\right) \in A_{a}}\{f(a) \wedge f(a)\} \\
\leq & \bigvee_{\left(\left(a\left(x^{2} a\right)\right), a\right) \in A_{a}}\left\{f\left(\left(a\left(x^{2} a\right)\right) a\right)\right\}=f(a) .
\end{aligned}
$$

Thus $(f \circ S) \circ f=f$.

Again by using left invertive law, medial law with left identity and paramedial law, we have

$$
\begin{aligned}
a & \leq(a a) x=(x a) a=(x((a a) x)) a=((a a)(x x)) a=(((x x) a) a) a \\
& =(((x x)((a a) x)) a) a=(((x x)((x a) a)) a) a \\
& =(((x x)((a e)(a x))) a) a=(((x x)(a((a e) x))) a) a \\
& =((a((x x)((a e) x))) a) a
\end{aligned}
$$

Therefore $(((a((x x)((a e) x))) a), a) \in A_{a}$, since $A_{a} \neq \emptyset$. Thus

$$
\begin{aligned}
(f \circ f)(a) & =\bigvee_{(((a((x x)((a e) x))) a), a) \in A_{a}}\{f((a((x x)((a e) x))) a) \wedge f(a)\} \\
& \geq\{f((a((x x)((a e) x))) a) \wedge f(a)\} \\
& \geq f(a) \wedge f(a) \wedge f(a)=f(a)
\end{aligned}
$$


thus by using Lemma $8, f \circ f=f$.

$($ ii $) \Longrightarrow(i)$ : Let $f$ be a fuzzy subset of a left regular $S$, then

$$
\begin{aligned}
f((x y) z) & =((f \circ S) \circ f)((x y) z) \\
& =\bigvee_{((x y), z) \in A_{(x y) z}}\{(f \circ S)(x y) \wedge f(z)\} \\
& \geq \bigvee_{(x, y) \in A_{x y}}\{f(x) \wedge S(y)\} \wedge f(z) \\
& \geq f(x) \wedge 1 \wedge f(z) \\
& =f(x) \wedge f(z) .
\end{aligned}
$$

Since $f \circ f=f$, therefore by Lemma $8, f$ is a fuzzy AG-subgroupoid of $S$. This shows that $f$ is a fuzzy bi ideal of $S$.

Theorem 5. In a left regular $S$ with left identity, the following statements are equivalent.

(i) $f$ is a fuzzy interior ideal of $S$.

(ii) $(S \circ f) \circ S=f$.

Proof. It is simple.

Theorem 6. In a left regular $S$ with left identity, the following statements are equivalent.

(i) $f$ is a fuzzy $(1,2)$-ideal of $S$.

(ii) $(f \circ S) \circ(f \circ f)=f$ and $f \circ f=f$.

Proof. (i) $\Longrightarrow$ (ii) : Let $f$ be a fuzzy $(1,2)$-ideal of a left regular $S$ with left identity and let $a \in S$, then there exists $x \in S$ such that $a \leq a^{2} x$. Now by using left invertive law and medial law with left identity, we have

$$
\begin{aligned}
a & \leq(a a) x=(x a) a=(x a)((a a) x)=(a a)((x a) x) \\
& =(a((a a) x))((x a) x)=((a a)(a x))((x a) x) \\
& =(((x a) x)(a x))(a a)=(a(((x a) x) x))(a a) .
\end{aligned}
$$

Thus $((a(((x a) x) x)), a a) \in A_{a}$, since $A_{a} \neq \emptyset$, therefore

$$
((f \circ S) \circ(f \circ f))(a)=\bigvee_{((a(((x a) x) x)), a a) \in A_{a}}\{(f \circ S)(a(((x a) x) x)) \wedge(f \circ f)(a a)\} .
$$

Now

$$
\begin{aligned}
(f \circ S)(a(((x a) x) x)) & =\bigvee_{(a,(((x a) x) x)) \in A_{a(((x a) x) x)}}\{f(a) \wedge S(((x a) x) x)\} \\
& \geq f(a) \wedge S(((x a) x) x)=f(a)
\end{aligned}
$$

and

$$
(f \circ f)(a a)=\bigvee_{(a, a)=A_{a a}}\{f(a) \wedge f(a)\} \geq f(a) .
$$

Thus we get

$$
((f \circ S) \circ(f \circ f))(a) \geq f(a) .
$$

Now by using medial law with left identity, left invertive law and paramedial law, we have

$$
\begin{aligned}
a & \leq(a a) x=(((a a) x)((a a) x)) x=((a a)(((a a) x) x)) x \\
& =((a a)((x x)(a a))) x=\left((a a)\left(x^{2}(a a)\right)\right) x \\
& =\left(x\left(x^{2}(a a)\right)\right)(a a)=\left(x\left(a\left(x^{2} a\right)\right)\right)(a a) \\
& =\left(a\left(x\left(x^{2} a\right)\right)\right)(a a)=\left(a\left(x\left(x^{2}((a a) x)\right)\right)\right)(a a) \\
& =\left(a\left(x\left((a a) x^{3}\right)\right)\right)(a a) .
\end{aligned}
$$


Therefore $\left(\left(a\left(x\left((a a) x^{3}\right)\right)\right), a a\right) \in A_{a}$, since $A_{a} \neq \emptyset$. Thus

$$
((f \circ S) \circ(f \circ f))(a)=\bigvee_{\left(\left(a\left(x\left((a a) x^{3}\right)\right)\right), a a\right) \in A_{a}}\left\{(f \circ S)\left(a\left(x\left((a a) x^{3}\right)\right)\right) \wedge(f \circ f)(a a)\right\}
$$

Now

$$
\begin{aligned}
(f \circ S)\left(a\left(x\left((a a) x^{3}\right)\right)\right) & =\bigvee_{\left(a,\left(x\left((a a) x^{3}\right)\right)\right) \in A_{a\left(x\left((a a) x^{3}\right)\right)}}\left\{f(a) \wedge S\left(x\left((a a) x^{3}\right)\right)\right\} \\
& =\bigvee_{\left(a,\left(x\left((a a) x^{3}\right)\right)\right) \in A_{a\left(x\left((a a) x^{3}\right)\right)}} f(a)
\end{aligned}
$$

and

$$
\begin{aligned}
(f \circ f)(a a) & =\bigvee_{(a, a) \in A_{a a}}\{f(a) \wedge f(a)\} \\
& =\bigvee_{(a, a) \in A_{a a}} f(a)
\end{aligned}
$$

Therefore

$$
\begin{aligned}
(f \circ S)\left(a\left(x\left((a a) x^{3}\right)\right)\right) \wedge(f \circ f)(a a) & =\bigvee_{\left(a,\left(x\left((a a) x^{3}\right)\right)\right) \in A_{\left.a\left(x(a a) x^{3}\right)\right)}} f(a) \wedge \bigvee_{(a, a) \in A_{a a}} f(a) \\
& =\bigvee_{\left(a,\left(x\left((a a) x^{3}\right)\right)\right) \in A_{\left.a\left(x((a)) x^{3}\right)\right)}}\{f(a) \wedge f(a)\} .
\end{aligned}
$$

Thus from above, we get

$$
\begin{aligned}
((f \circ S) \circ(f \circ f))(a) & =\bigvee_{\left(\left(a\left(x\left((a a) x^{3}\right)\right)\right), a a\right) \in A_{a}}\left\{\bigvee_{\left(a,\left(x\left((a a) x^{3}\right)\right)\right) \in A_{a\left(x\left((a) x^{3}\right)\right)}} f(a)\right\} \\
& =\bigvee_{\left(\left(a\left(x\left((a a) x^{3}\right)\right)\right), a a\right) \in A_{a}}\{f(a) \wedge f(a) \wedge f(a)\} \\
& \leq \bigvee_{\left(\left(a\left(x\left((a a) x^{3}\right)\right)\right), a a\right) \in A_{a}} f\left(\left(a\left(x\left((a a) x^{3}\right)\right)\right)(a a)\right)=f(a) .
\end{aligned}
$$

Therefore $(f \circ S) \circ(f \circ f)=f$.

Now by using left invertive law and medial law with left identity, we have

$$
\begin{aligned}
a & \leq(a a) x=(x a) a=(x((a a) x)) a=((a a)(x x)) a \\
& =\left((a((a a) x)) x^{2}\right) a=\left(((a a)(a x)) x^{2}\right) a \\
& =\left(\left(x^{2}(a x)\right)(a a)\right) a=\left(\left(a x^{3}\right)(a a)\right) a .
\end{aligned}
$$

Thus $\left(\left(\left(a x^{3}\right)(a a)\right), a\right) \in A_{a}$, since $A_{a} \neq \emptyset$, therefore

$$
\begin{aligned}
(f \circ f)(a) & =\bigvee_{\left(\left(\left(a x^{3}\right)(a a)\right), a\right) \in A_{a}}\left\{f\left(\left(\left(a x^{3}\right)(a a)\right)\right) \wedge f(a)\right\} \\
& \geq f(a) \wedge f(a) \wedge f(a)=f(a) .
\end{aligned}
$$

Now by using Lemma $8, f \circ f=f$.

(ii) $\Longrightarrow($ i $)$ : Let $f$ be a fuzzy subset of a left regular $S$. Now since $f \circ f=f$, therefore by Lemma $8, f$ is a fuzzy 
LA-subsemigroup of $S$

$$
\begin{aligned}
f((x a)(y z)) & =((f \circ S) \circ(f \circ f))((x a)(y z)) \\
& =((f \circ S) \circ f)((x a)(y z)) \\
& =\bigvee_{((x a),(y z)) \in A_{(x a)(y z)}}\{(f \circ S)(x a) \wedge f(y z)\} \\
& \geq(f \circ S)(x a) \wedge f(y z) \\
& =\bigvee_{(x, a) \in A_{(x a)}}\{f(x) \wedge S(a)\} \wedge f(y z) \\
& \geq f(x) \wedge 1 \wedge f(y) \wedge f(z) \\
& =f(x) \wedge f(y) \wedge f(z) .
\end{aligned}
$$

This shows that $f((x a)(y z)) \geq f(x) \wedge f(y) \wedge f(z)$, therefore $f$ is a fuzzy (1,2)-ideal of $S$.

The proof of following two Lemmas are same as in (Kehayapulu, N. 2006) and (Xie, X. Y. 2008) respectively.

Lemma 9. The following properties hold in $S$.

(i) A non-empty subset $A$ of $S$ is an $A G$-subgroupoid of $S$ if and only if $C_{A}$ is a fuzzy AG-subgroupoid of $S$.

(ii) A a non-empty subset $A$ of $S$ is left (right,two-sided) ideal of $S$ if and only if $C_{A}$ is a fuzzy left (right,two-sided) ideal of $S$.

(iii) For the non-empty sets $A$ and $B$ of $S, C_{A} \cap C_{B}=C_{A \cap B}$ and $C_{A} \circ C_{B}=C_{(A B]}$.

Lemma 10. For all $A, B \subseteq S,(A](B] \subseteq(A B]$ holds.

Lemma 11. For all $a \in S$ with left identity, $(S a]$ and $\left(a^{2} S\right]$ are left and right ideals of $S$ respectively.

Proof. Let $a$ be any element of $S$ with left identity and consider an element $x$ in $S(S a$ ], then $x=y z$ for some $y$ in $S$ and $z$ in $(S a]$, where $z \leq s a$ for some $s a$ in $S a$. Now by using paramedial law and left invertive law, we have

$$
x \leq y(s a)=(e y)(s a)=(a s)(y e)=((y e) s) a,
$$

where $(y e) s)$ is in $S$, which implies that $x$ is in $(S a$. Assume that $y$ be any element of $S$ and let $y \leq x$ such that $x$ is in $(S a]$, then $y \leq x \leq s a$ for some $s a$ in $S a$. This implies that $y$ is in $(S a$. Hence $(S a]$ is a left ideal of $S$.

Similarly we can show that $\left(a^{2} S\right]$ is a right ideal of $S$.

Theorem 7. For $S$ with left identity, the following conditions are equivalent.

(i) $S$ is left regular.

(ii) $L \cap R \subseteq(L R]$, for every left ideal $L$ and every right $R$ of $S$, where a right ideal $R$ is semiprime.

(iii) $f \cap g \subseteq f \circ g$, for every fuzzy left ideal $f$ and every fuzzy right ideal $g$ of $S$, where a fuzzy right ideal $g$ is fuzzy semiprime.

Proof. (i) $\Longrightarrow$ (iii) : Assume that $S$ is left regular. Let $f$ and $g$ be any fuzzy left and fuzzy right ideal of $S$ with left identity. For each $a \in S$ there exists $x \in S$ such that $a \leq x a^{2}$, then by using medial law with left identity, we have

$$
a \leq x(a a)=a(x a) \leq a(x(x(a a)))=a(x(a(x a)))=a(a(x(x a))) .
$$

Thus $(a,(a(x(x a)))) \in A_{a}$, since $A_{a} \neq \emptyset$, therefore

$$
\begin{aligned}
(f \circ g)(a) & =\bigvee_{(a,(a(x(x a)))) \in A_{a}}\{f(a) \wedge g(a(x(x a)))\} \\
& \geq f(a) \wedge g(a)=(f \cap g)(a) .
\end{aligned}
$$

Therefore $f \cap g \subseteq f \circ g$, and by using paramedial law, we have

$$
a \leq x(a a)=(e x)(a a)=(a a)(x e)=a^{2}(x e)
$$


therefore

$$
g(a) \geq g\left(a^{2}(x e)\right) \geq g\left(a^{2}\right) \text {. Hence } g \text { is a fuzzy semiprime. }
$$

(iii) $\Longrightarrow$ (ii) : Assume that $L$ and $R$ be any left and right ideal of $S$, then by Lemma 9 (ii), $C_{L}$ and $C_{R}$ are fuzzy left and fuzzy right ideals of $S$ respectively. Let $a \in L \cap R$, then $a \in L$ and $a \in R$, therefore by Lemma 9 (iii) and given assumption, we have

$$
C_{L \cap R}(a)=\left(C_{L} \cap C_{R}\right)(a) \leq\left(C_{L} \circ C_{R}\right)(a)=\left(C_{(L R]}\right)(a)=1
$$

Hence $L \cap R \subseteq(L R]$. Let $a^{2} \in R$, then by assumption, the characteristic function $C_{R}$ of a right ideal $R$ is fuzzy semiprime, therefore

$$
C_{R}(a) \geq C_{R}\left(a^{2}\right)=1
$$

which implies that $a \in R$, thus $R$ is semiprime.

$($ ii $) \Longrightarrow(i)$ : Let $a \in S$ with left identity, then clearly $a \in(S a]$ and $a^{2} \in\left(a^{2} S\right]$. From Lemma $11,(S a]$ and $\left(a^{2} S\right]$ are left and right ideals of $S$ respectively. By assumption, every right ideal of $S$ is semiprime, which implies that $a \in\left(a^{2} S\right]$, thus by using Lemma 10, medial law, paramedial law and left invertive law, we have

$$
\begin{aligned}
a & \in(S a] \cap\left(a^{2} S\right] \subseteq(S a]\left(a^{2} S\right] \subseteq\left((S a)\left(a^{2} S\right)\right]=\left(\left(S a^{2}\right)(a S)\right] \\
& =(((e S)(a a))(a S)]=(((a a)(S e))(a S)] \subseteq\left(\left(a^{2} S\right)(a S)\right] \\
& =\left(\left(((a S) S) a^{2}\right)\right] \subseteq\left(S a^{2}\right] .
\end{aligned}
$$

Hence $S$ is left regular.

Theorem 8. For $S$ with left identity, the following conditions are equivalent.

(i) $S$ is left regular.

(ii) $f \cap g=f \circ g$, for every fuzzy right ideal $f$ and every fuzzy left ideal $g$ of $S$, where a fuzzy right ideal $f$ is fuzzy semiprime.

Proof. (i) $\Longrightarrow$ (ii) : Assume that $S$ is left regular. Let $f$ and $g$ be any fuzzy right and fuzzy left ideals of $S$ respectively, then clearly $f \circ g \subseteq f \cap g$. Since $S$ is left regular, therefore for each $a \in S$ there exists $x \in S$ such that $a \leq x a^{2}$. Now by using medial law with left identity, paramedial law and left invertive law, we have

$$
a \leq x(a a)=a(x a)=(e a)(x a)=(a x)(a e)=((a e) x) a .
$$

Thus (((ae)x),a) $\in A_{a}$, since $A_{a} \neq \emptyset$, therefore

$$
\begin{aligned}
(f \circ g)(a) & =\bigvee_{(((a e) x), a) \in A_{a}}\{f((a e) x) \wedge g(a)\} \geq f((a e) x) \wedge g(a) \\
& \geq f(a) \wedge g(a)=(f \cap g)(a) .
\end{aligned}
$$

Now by using paramedial law, we have

$$
a \leq(e x)(a a)=(a a)(x e)=a^{2}(x e)
$$

therefore

$$
f(a) \geq f\left(a^{2}(x e)\right) \geq f\left(a^{2}\right) \text {. Hence } f \text { is a fuzzy semiprime. }
$$

(ii) $\Longrightarrow\left(\right.$ (i) : Assume that $R$ and $L$ be any right and left ideals of $S$ respectively, then by Lemma 9 (ii), $C_{R}$ and $C_{L}$ are fuzzy right and fuzzy left ideal of $S$ respectively. Let $a \in R \cap L$, then $a \in R$ and $a \in L$. Therefore by Lemma 9 (iii) and given assumption, we have

$$
C_{R \cap L}(a)=\left(C_{R} \cap C_{L}\right)(a)=\left(C_{R} \circ C_{L}\right)(a)=\left(C_{(R L]}\right)(a)=1,
$$

which implies that $R \cap L \subseteq(R L]$. Now by assumption $C_{R}$ is a fuzzy semiprime, then it is easy to see that $R$ is semiprime. From Lemma 11, $(S a]$ and $\left(a^{2} S\right]$ are left and right ideals of $S$ respectively such that $a \in(S a]$ and $a^{2} \in\left(a^{2} S\right]$. Therefore by using Lemma 10 and left invertive law, we have

$$
a \in\left(a^{2} S\right] \cap(S a] \subseteq\left(a^{2} S\right](S a] \subseteq\left(\left(a^{2} S\right)(S a)\right]=\left(((S a) S) a^{2}\right] \subseteq\left(S a^{2}\right] .
$$

Hence $S$ is left regular. 
Theorem 9. In $S$ with left identity, the following conditions are equivalent.

(i) $S$ is left regular.

(ii) $R \cap L=(R L]$ for every right ideal $R$ and every left ideal $L$ of $S$, where a right ideal $R$ is semiprime.

Proof. It is straightforward.

\section{References}

Chang, C. L. (1968). Fuzzy topological spaces. J. Math. Anal. Appl., 24, $182-190$.

Holgate, P. (1992). Groupoids satisfying a simple invertive law. The Math. Stud., 61, 101 - 106.

Kazim, M. A. and Naseeruddin, M. (1972). On almost semigroups. The Alig. Bull. Math., 2, 1 - 7.

Kehayapulu, N. and Tsingelis, M. (2002). Fuzzy sets in ordered groupoids. Semigroups Forum, 5, 28 - 132.

Kehayapulu, N. and Tsingelis, M. (2006). Regular ordered semigroups in terms of fuzzy subsets. Inform. Sci., 176, $3675-3693$.

Khan, M. (2008). Some studies in AG*-groupoids. Ph. D., thesis, Quaid-i-Azam University, Islamabad, Pakistan.

Khan, M. and Khan, M. N. A. (2010). On fuzzy Abel-Grassmann's groupoids. Advances in Fuzzy Mathematics, 3 , $349-360$.

Kuroki, N. (1979). Fuzzy bi-ideals in Semigroups. Comment. Math. Univ. St. Pauli, 27, 17 - 21.

Mordeson, J. N. (2003). Fuzzy semigroups. Springer-Verlag, Berlin.

Mushtaq, Q. and Yusuf, S. M. (1978). On LA-semigroups. The Alig. Bull. Math., 8, 65 - 70.

Mushtaq, Q. and Yusuf, S. M. (1988). On LA-semigroup defined by a commutative inverse semigroup. Math. Bech., 40, $59-62$.

Protic, P. V. and Stevanović, N. (1994). On Abel-Grassmann's groupoids. Proc. Conf. Pristina, 31 - 38.

Rosenfeld, A. (1971). Fuzzy groups. J. Math. Anal. Appl., 35, 512 - 517.

Stevanović, N. and Protić, P. V. (2004). Composition of Abel-Grassmann's 3-bands. Novi Sad, J. Math., $2,34,175$ - 182.

Xie, X. Y. and, Tang, J. (2008). Fuzzy radicals and prime fuzzy ideals of ordered semigroups. Inform. Sci., 178, $4357-4374$.

Zadeh, L. A. (1965). Fuzzy sets. Inform. Control, 8, 338 - 353. 